\title{
CONTROL OF A QUANTUM PARTICLE IN A MOVING POTENTIAL WELL
}

\author{
Pierre Rouchon*
}

\author{
* Ecole des Mines de Paris, Centre Automatique et \\ Systèmes, 60 Bd Saint-Michel, 75272 Paris cedex 06 \\ FRANCE. (pierre.rouchon@ensmp.fr)
}

\begin{abstract}
The control is the potential well absolute position. For two kinds of potential shape (periodic and box), we propose approximated solutions to the stead-state motion planing problem: steering in finite time the particle from an initial well position to a final well position, the initial and final particle energies being identical. This problem is a quantum analogue of the water tank problem, where a tank filled with liquid is moved from one position where the surface is horizontal to another position where the surface is also horizontal.

Copyright (C) 2003 IFAC
\end{abstract}

Keywords: Quantum system, controllability, motion planning, operational calculus, Gevrey functions.

\section{INTRODUCTION}

We consider the control of a quantum particle represented by a probability complex amplitude $\mathbb{R} \ni q \mapsto \psi(q, t)$ solution of

$$
\imath \frac{\partial \psi}{\partial t}=-\frac{1}{2} \frac{\partial^{2} \psi}{\partial q^{2}}+(V(q)+\ddot{v} q) \psi
$$

This 1-D Schrödinger equation describes the non relativistic motion of a single charged particle (mass $m=1, \hbar=1$ ) with a potential $V$ in a non Galilean frame $z$ of absolute position $v$, corresponding to the position of the well. Changes of independent variables $(t, q) \mapsto(t, z)$ and dependent variable $\psi \mapsto \phi$, transform (1) into (2) where the control appears as a shift on the space variable. These classical transformations are as follows (see, e.g., (Butkovskiy and Samoilenko, 1984)). The transformations $q=z-v$ and

$\psi(t, z-v)=\exp \left(\imath\left(-z \dot{v}-v \dot{v}+\frac{1}{2} \int_{0}^{t} \dot{v}^{2}\right)\right) \phi(t, z)$

yield

$$
\imath \frac{\partial \phi}{\partial t}=-\frac{1}{2} \frac{\partial^{2} \phi}{\partial z^{2}}+V(z-v) \phi
$$

corresponding to the Schrödinger equation in a Galilean frame $q$.

Controllability depends strongly on the shape of the potential $V$. We will discuss here some preliminary results with the following potential shape.

- The periodic potential, $V(q)=V(q+a)$, where impulsive controls achieve iso-energy translations with amplitudes multiple of the period $a$.

- The box potential (see figure 1$), V(q)=$ 0 for $q \in[-1 / 2,1 / 2]$ and $V(q)=+\infty$ for $q$ outside $[-1 / 2,1 / 2]$. This problem admits strong similarity with the water tank problem: around any state of definite energy, the linear tangent approximate system is not controllable but it is "steady-state" controllable in the sense of (Petit and Rouchon, 2002). We guess that, as for the watertank system (Coron, 2002), the nonlinear dynamics is locally controllable around any state of definite energy. 


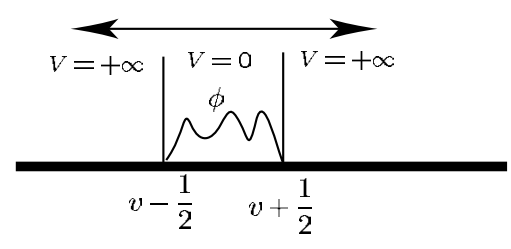

Fig. 1. Quantum particle in a moving box

\section{PERIODIC POTENTIAL}

Take (1) with a periodic potential $(\operatorname{period} a>0)$ :

$$
V(q+a)=V(q), \forall q .
$$

The goal is to solve approximatively the transition between two bounded states of the same energy $\psi_{1}$ and $\psi_{2}$ such that

$$
\psi_{2}(q)=\psi_{1}(q-k a)
$$

where $k \in \mathbb{Z}$.

Take the form (2) with $v$ as control. Take any $C^{2}$ function $[0,1] \ni \alpha \mapsto y(\alpha) \in \mathbb{R}$ such that

$$
y(0)=\dot{y}(0)=\ddot{y}(0)=\dot{y}(1)=\ddot{y}(1), \quad y(1)=k a
$$

Then, for $\varepsilon>0$ small enough the control

$$
[0, T] \ni t \mapsto v(t)= \begin{cases}0 & \text { for } t<0 \\ y(t / \varepsilon) & \text { for } t \in[0, \varepsilon] \\ a & \text { for } t>\varepsilon .\end{cases}
$$

steers, approximatively, from $\psi_{1}$ to $\psi_{2}$. This is obvious with $(2): v(t)$ is close to a step between 0 and $k a$; since $V(z-k a)=V(z)$, the influence of such variation of $v$ on $\phi$ solution of (2) remains small $(O(\varepsilon))$. Thus $\phi$ remains closed to $\psi_{1}(z)$ during the impulse. Thus, up to a phase shift the real state $\psi(\varepsilon, q)$ corresponds to $\phi(\varepsilon, z)=\psi_{1}(z)=$ $\psi_{1}(q-k a)=\psi_{2}(q)$.

This simple impulsive control overcomes the following difficulty: such transitions necessarily requires to reach energies in the continuous part of the spectrum. Moreover straightforward extensions to $2 D$ or $3 D$ periodic potentials can be done.

\section{THE MOVING BOX}

As illustrated on figure 1 take (2), with $V(z)=0$ for $z \in\left[-\frac{1}{2}, \frac{1}{2}\right]$ and $V(z)=+\infty$ for $z$ outside $\left[-\frac{1}{2}, \frac{1}{2}\right]$. The dynamics reads:

$$
\begin{aligned}
\imath \frac{\partial \phi}{\partial t} & =-\frac{1}{2} \frac{\partial^{2} \phi}{\partial z^{2}}, \quad z \in\left[v-\frac{1}{2}, v+\frac{1}{2}\right], \\
\phi\left(v-\frac{1}{2}, t\right) & =\phi\left(v+\frac{1}{2}, t\right)=0
\end{aligned}
$$

where $v$ is the position of the box and $z$ is an absolute position (Galilean frame). Otherwise stated (see (1))

$$
\begin{aligned}
\imath \frac{\partial \psi}{\partial t} & =-\frac{1}{2} \frac{\partial^{2} \psi}{\partial q^{2}}+\ddot{v} q \psi, \quad q \in\left[-\frac{1}{2}, \frac{1}{2}\right], \\
\psi\left(-\frac{1}{2}, t\right) & =\psi\left(\frac{1}{2}, t\right)=0
\end{aligned}
$$

where $q=z-v$ is the relative position with respect to the box. $\psi$ and $\phi$ are related via

$\psi(t, z-v)=\exp \left(\imath\left(-z \dot{v}-\nu \dot{v}+\frac{1}{2} \int_{0}^{t} \dot{v}^{2}\right)\right) \phi(t, z)$.

\subsection{Modal decomposition}

For $v=0$, the system admits a non-degenerate discrete spectrum (see, e.g.,(Messiah, 1962)):

$$
\begin{aligned}
\omega_{2 n} & =2 n^{2} \pi^{2} \\
\psi_{2 n}(q) & =2 \sin (2 n \pi q) \\
\omega_{2 n+1} & =2\left(n+\frac{1}{2}\right)^{2} \pi^{2} \\
\psi_{2 n+1}(q) & =2 \cos ((2 n+1) \pi q)
\end{aligned}
$$

Set $\psi(t, q)=\sum_{n \geq 1} a_{n}(t) \psi_{n}(q)$ in $\imath \frac{\partial \psi}{\partial t}=-\frac{1}{2} \frac{\partial^{2} \psi}{\partial q^{2}}+$ $\ddot{v} q \psi$, to obtain, for each integer $n \geq 1$,

$$
\begin{aligned}
\imath \frac{d}{d t} a_{2 n}= & -\omega_{2 n} a_{2 n} \\
& +\ddot{v}\left(\sum_{k \geq 0} a_{2 k+1} \int_{-\frac{1}{2}}^{\frac{1}{2}} q \psi_{2 n}(q) \psi_{2 k+1}(q) d q\right) \\
\imath \frac{d}{d t} a_{2 n+1}= & -\omega_{2 n+1} a_{2 n+1} \\
& +\ddot{v}\left(\sum_{k \geq 1} a_{2 k} \int_{-\frac{1}{2}}^{\frac{1}{2}} q \psi_{2 n+1}(q) \psi_{2 k}(q) d q\right) .
\end{aligned}
$$

For any integers $\alpha \geq 1$ and $\beta \geq 0$ :

$$
\begin{aligned}
& \int_{-\frac{1}{2}}^{\frac{1}{2}} q \psi_{2 \alpha}(q) \psi_{2 \beta+1}(q) d q= \\
& \quad(-1)^{\alpha+\beta}\left(\frac{1}{\left[\left(\alpha+\beta+\frac{1}{2}\right) \pi\right]^{2}}+\frac{1}{\left[\left(\alpha-\beta-\frac{1}{2}\right) \pi\right]^{2}}\right)
\end{aligned}
$$

Notice that odd (resp. even) modes are connected via the control $v$ to even (resp. odd) modes (selection rules).

\subsection{Tangent linearization}

Denote by $\bar{\psi}$ any state of definite energy $\bar{\omega}$ in $(3)$ or (4). Set

$$
\psi(t, q)=\exp (-\imath \bar{\omega} t)(\bar{\psi}(q)+\Psi(q, t))
$$

in (2). Then $\Psi$ satisfies

$$
\begin{aligned}
\imath \frac{\partial \Psi}{\partial t}+\bar{\omega} \Psi & =-\frac{1}{2} \frac{\partial^{2} \Psi}{\partial q^{2}}+\ddot{v} q(\bar{\psi}+\Psi) \\
0 & =\Psi\left(-\frac{1}{2}, t\right)=\Psi\left(\frac{1}{2}, t\right) .
\end{aligned}
$$

The tangent linear system is obtained, assuming $\Psi$ and $\ddot{v}$ small and neglecting the second order term $\ddot{v} q \Psi$ :

$$
\begin{aligned}
& \imath \frac{\partial \Psi}{\partial t}+\bar{\omega} \Psi=-\frac{1}{2} \frac{\partial^{2} \Psi}{\partial q^{2}}+\ddot{v} q \bar{\psi} \\
& \Psi\left(-\frac{1}{2}, t\right)=\Psi\left(\frac{1}{2}, t\right)=0
\end{aligned}
$$


We prove here below via operational computations that (5) is not controllable but steady-state controllable. We give explicit formulae for the control $[0, T] \ni t \mapsto \ddot{v}(t)$, steering in finite time from $\Psi=0, v=\dot{v}=0$ at $t=0$ to $\Psi=0, v=a, \dot{v}=0$ at $t=T$ for any $T>0$. Computations are similar to those we have proposed for heat or EulerBernouilli dynamics where ultra-distributions and Gevrey functions of order $\leq 1$ appear (Laroche et al., 2000; Fliess et al., 1996; Rouchon, 2001).

Set $s=d / d t$. Standard computations show that the general solution of

is

$$
(\imath s+\bar{\omega}) \Psi=-\frac{1}{2} \Psi^{\prime \prime}+s^{2} v q \bar{\psi}
$$

$$
\Psi=A(s, q) a(s)+B(s, q) b(s)+C(s, q) v(s)
$$

where

$$
\begin{aligned}
A(s, q) & =\cos (q \sqrt{2 \imath s+2 \bar{\omega}}) \\
B(s, q) & =\frac{\sin (q \sqrt{2 \imath s+2 \bar{\omega}})}{\sqrt{2 \imath s+2 \bar{\omega}}} \\
C(s, q) & =\left(-\imath s q \bar{\psi}(q)+\bar{\psi}^{\prime}(q)\right) .
\end{aligned}
$$

\subsection{Case $q \mapsto \bar{\phi}(q)$ even.}

The boundary conditions imply

$A(s, 1 / 2) a(s)=0, \quad B(s, 1 / 2) b(s)=-\psi^{\prime}(1 / 2) v(s)$.

The element $a(s)$ is a torsion element (Mounier, 1998), thus the system is not controllable. Nevertheless, for steady-state controllability, we have $a \equiv 0$ (as for the water tank (Petit and Rouchon, 2002)) and we have the following parameterization $^{1}$ :

$$
\begin{aligned}
b(s) & =-\bar{\psi}^{\prime}(1 / 2) \frac{\sin \left(\frac{1}{2} \sqrt{-2 \imath s+2 \bar{\omega}}\right)}{\sqrt{-2 \imath s+2 \bar{\omega}}} y(s) \\
v(s) & =\frac{\sin \left(\frac{1}{2} \sqrt{2 \imath s+2 \bar{\omega}}\right)}{\sqrt{2 \imath s+2 \bar{\omega}}} \frac{\sin \left(\frac{1}{2} \sqrt{-2 \imath s+2 \bar{\omega}}\right)}{\sqrt{-2 \imath s+2 \bar{\omega}}} y(s) \\
\Psi(s, q) & =B(s, q) b(s)+C(s, q) v(s)
\end{aligned}
$$

The entire functions of $s$ appearing in this formulae are of order less than $1 / 2$, i.e., their module for $s$ large is bounded by $\exp (M \sqrt{|s|})$ for some $M>0$, independent of $s \in \mathbb{C}$ and $q \in[-1,1]$. The above formulae (6) admit then a clear interpretation in the time domain, as for the heat equation with the Holmgren series solution (Valiron, 1950), when $y$ is a $C^{\infty}$ time function of Gevrey order less than 1: i.e. $\exists M>0$ and $\exists \sigma \in[0,1]$ such that, $\forall t, \forall n,\left|y^{(n)}(t)\right| \leq M^{n} \Gamma(1+(\sigma+1) n)$ where $\Gamma$ is the Gamma function ${ }^{2}$. This results from the following fact: to an entire function of $s, F(s)$, of order $\leq 1 / 2$ is associated a series $\sum_{n \geq 0} a_{n} s^{n}$ with

\footnotetext{
1 Remember that $v$ is associated to a real quantity and the operator $\frac{\sin \left(\frac{1}{2} \sqrt{2 \imath s+2 \bar{\omega}}\right)}{\sqrt{2 \imath s+2 \bar{\omega}}} \frac{\sin \left(\frac{1}{2} \sqrt{-2 \imath s+2 \bar{\omega}}\right)}{\sqrt{-2 \imath s+2 \bar{\omega}}}$ is a real operator. 2 Analytic functions are Gevrey functions of order $\sigma=0$.
}

coefficient $a_{n}$ satisfying $\left|a_{n}\right| \leq K^{n} / \Gamma(1+2 n)$ for all $n$, with some constant $K>0$ independent of $n$. To $F(s) y(s)$ corresponds in the time domain, the series,

$$
\sum_{n \geq 0} a_{n} y^{(n)}(t)
$$

that is absolutely convergent when $y$ is a Gevrey function of order $\sigma<1$. Take $T>0$ and $D \in \mathbb{R}$. Steering (5) from $\Psi=0, v=0$ at time $t=0$, to $\Psi=0, v=D$ at $t=T$ is possible with the following Gevrey function of order $\sigma$ :

$$
[0, T] \ni t \mapsto y(t)=
$$

$\begin{cases}0 & \text { for } t \leq 0 \\ \bar{D} \frac{\exp \left(-\left(\frac{T}{t}\right)^{\frac{1}{\sigma}}\right)}{\exp \left(-\left(\frac{T}{t}\right)^{\frac{1}{\sigma}}\right)+\exp \left(-\left(\frac{T}{T-t}\right)^{\frac{1}{\sigma}}\right)} & \text { for } 0<t<T \\ \bar{D} & \text { for } t \geq T\end{cases}$

with $\bar{D}=\frac{2 \bar{\omega} D}{\sin ^{2}(\sqrt{\bar{\omega} / 2})}$. The fact that this function is of Gevrey order $\sigma$ results from its exponential decay of order $\sigma$ around 0 and 1 (see, e.g., (Ramis, 1993; Ramis, 1978)).

\subsection{Case $q \mapsto \bar{\phi}(q)$ odd.}

The boundary conditions imply

$B(s, 1 / 2) b(s)=0, \quad A(s, 1 / 2) a(s)=-\psi^{\prime}(1 / 2) v(s)$.

$b$ is a torsion element and thus the system is not controllable. Nevertheless, as for the even case, we have the following parameterization:

$$
\begin{aligned}
a(s) & =-\bar{\psi}^{\prime}(1 / 2) \cos \left(\frac{1}{2} \sqrt{-2 \imath s+2 \bar{\omega}}\right) y(s) \\
v(s) & =\cos \left(\frac{1}{2} \sqrt{2 \imath s+2 \bar{\omega}}\right) \cos \left(\frac{1}{2} \sqrt{-2 \imath s+2 \bar{\omega}}\right) y(s) \\
\Psi(s, q) & =A(s, q) a(s)+C(s, q) v(s) .
\end{aligned}
$$

As for the even case, with

$[0, T] \ni t \mapsto y(t)=$

$$
\begin{cases}0 & \text { for } t \leq 0 \\ \bar{D} \frac{\exp \left(-\left(\frac{T}{t}\right)^{\frac{1}{\sigma}}\right)}{\exp \left(-\left(\frac{T}{t}\right)^{\frac{1}{\sigma}}\right)+\exp \left(-\left(\frac{T}{T-t}\right)^{\frac{1}{\sigma}}\right)} & \text { for } 0<t<T \\ \bar{D} & \text { for } t \geq T\end{cases}
$$

where $\bar{D}=\frac{D}{\cos ^{2}(\sqrt{\bar{\omega} / 2})}$, we can steer (5) from $\Psi=0, v=0$ at time $t=0$, to $\Psi=0, v=D$ at $t=T$.

\subsection{Practical computations}

The above method for computing the steering control requires to develop in series of $s$ and to calculate high order time derivatives of $y$. All these calculations can be bypassed with Cauchy 
formula. Take a bounded measurable function $t \mapsto Y(t)$ corresponding to the position set-point for $v$. From this function, we deduce a complex entire function $\zeta \mapsto y(\zeta)$ via convolution with a Gaussian kernel with standard deviation $\varepsilon$

$$
y(\zeta)=\frac{1}{\varepsilon \sqrt{2 \pi}} \int_{-\infty}^{+\infty} \exp \left(-\frac{(\zeta-t)^{2}}{2 \varepsilon^{2}}\right) Y(t) d t
$$

Consider, e.g, the relation giving the control $v$ in the even case: $v(s)=F(s) y(s)$ where

$$
F(s)=\frac{\sin \left(\frac{1}{2} \sqrt{2 \imath s+2 \bar{\omega}}\right)}{\sqrt{2 \imath s+2 \bar{\omega}}} \frac{\sin \left(\frac{1}{2} \sqrt{-2 \imath s+2 \bar{\omega}}\right)}{\sqrt{-2 \imath s+2 \bar{\omega}}}
$$

is an entire function of order less than 1 (order $1 / 2$ in fact but 1 is enough here). Thus $F(s)=$ $\sum_{n \geq 0} a_{n} s^{n}$ where $\left|a_{n}\right| \leq K^{n} / \Gamma(1+n)$ with $K>0$ independent of $n$. In the time domain $F(s) y(s)$ corresponds to $\sum_{n \geq 0} a_{n} y^{(n)}(t)$. But

$$
y^{(n)}(t)=\frac{\Gamma(n+1)}{2 \imath \pi} \oint_{\gamma} \frac{y(t+\xi)}{\xi^{n+1}} d \xi
$$

where $\gamma$ is a closed path around zero. Thus $\sum_{n \geq 0} a_{n} y^{(n)}(t)$ becomes

$$
\begin{array}{r}
\sum_{n \geq 0} a_{n} \frac{\Gamma(n+1)}{2 \imath \pi} \oint_{\gamma} \frac{y(t+\xi)}{\xi^{n+1}} d \xi= \\
\frac{1}{2 \imath \pi} \oint_{\gamma}\left(\sum_{n \geq 0} a_{n} \frac{\Gamma(n+1)}{\xi^{n+1}}\right) y(t+\xi) d \xi
\end{array}
$$

where ${ }^{3}$

$\sum_{n \geq 0} a_{n} \frac{\Gamma(n+1)}{\xi^{n+1}}=\int_{D_{\delta}} F(s) \exp (-s \xi) d s=B_{1}(F)(\xi)$.

is the Borel transform (see, e.g., (Boas, 1954)) of the $F$ that is defined for $\xi \in \mathbb{C}$ large enough, $|\xi|>K$. In the time domain $F(s) y(s)$ corresponds to

$$
\frac{1}{2 \imath \pi} \oint_{\gamma} B_{1}(F)(\xi) y(t+\xi) d \xi
$$

where $\gamma$ is a closed path around zero. Since $y(\zeta)=$ $\frac{1}{\varepsilon \sqrt{2 \pi}} \int_{-\infty}^{+\infty} \exp \left(-(\zeta-t)^{2} / 2 \varepsilon^{2}\right) Y(t) d t$ we have the following filter to deduce the control from the reference signal $Y(t)$ :

$$
\begin{aligned}
& v(t)= \\
& \int_{-\infty}^{+\infty}\left[\oint_{\gamma} \frac{B_{1}(F)(\xi)}{2 \varepsilon(2 \pi)^{\frac{3}{2}}} \exp \left(-\frac{(\xi-\tau)^{2}}{2 \varepsilon^{2}}\right) d \xi\right] Y(t-\tau) d \tau
\end{aligned}
$$

The kernel

$f(\tau)=\frac{1}{\imath \varepsilon(2 \pi)^{\frac{3}{2}}} \oint_{\gamma} B_{1}(F)(\xi) \exp \left(-(\xi-\tau)^{2} / 2 \varepsilon^{2}\right) d \xi$ can be computed numerically once for all. One can check that $f(\tau)$ is real and vanishes rapidly for $|\tau| \gg \varepsilon$. In fact $F$ is here of order $1 / 2$, thus $B_{1}(F)$ is defined on $\mathbb{C} /\{0\}$ and admits an essential singularity in 0 and Thus, the contour $\gamma$ is any

\footnotetext{
$3 D_{\delta}$ is the half line starting from 0 in the complex plane with direction $\delta$ chosen to ensure the convergence of the integral.
}

contour around 0. Such computations provide a simple numerical method to generate trajectories and to solve approximatively the steady-states motion planning problem for (5). These formulas are used in a Matlab animation that can be obtained upon request from the author.

\section{REFERENCES}

Boas, R.P. (1954). Entire functions. Academic Press, New York.

Butkovskiy, A.G. and Y.I. Samoilenko (1984). Control of Quantum-Mechnical Processes and Systems. Vol. 56 of Mathematics and its Applications (Soviet Series. Kluver Academic Publishers. Dortrecht/Boston/London. English edition 1990.

Coron, J.M. (2002). Local controllability of a 1-d tank containing a fluid modeled by the shallow water equations. ESAIM: $C O C V \mathbf{8}, 513-$ 554 .

Fliess, M., H. Mounier, P. Rouchon and J. Rudolph (1996). Systèmes linéaires sur les opérateurs de Mikusiński et commande d'une poutre flexible. In: ESAIM Proc. "Élasticité, viscolélasticité et contrôle optimal", sème entretiens du centre Jacques Cartier, Lyon. pp. 157-168.

Laroche, B., Ph. Martin and P. Rouchon (2000). Motion planing for the heat equation. Int. Journal of Robust and Nonlinear Control 10, 629-643.

Messiah, A. (1962). Quantum Mechanics. Vol. I\&II. North Holland Publ. Co.. Amsterdam.

Mounier, H. (1998). Algebraic interpretations of the spectral controllability of a linear delay system. Forum Math. 10, 39-58.

Petit, N. and P. Rouchon (2002). Dynamics and solutions to some control problems for watertank systems. IEEE AC 47(4), 594-609.

Ramis, J.P. (1978). Dévissage Gevrey. Astérisque 59-60, 173-204.

Ramis, J.P. (1993). Séries Divergentes et Théories Asymptotiques. Panoramas et synthèses. Société Mathématique de France.

Rouchon, P. (2001). Motion planning, equivalence, infinite dimensional systems. Int. J. Applied Mathematics and Computer Science 11(1), 165-188.

Schirmer, S. G., H. Fu and A. I. Solomon (2001). Complete controllability of quantum systems. Physical Review A.

Valiron, G. (1950). Equations Fonctionnelles. 2nd ed.. Masson et Cie, Editeurs, Paris. 